\title{
Fatores ecológicos determinantes na ocorrência de Araucaria angustifolia e Podocarpus lambertii, na Floresta Ombrófila Mista da FLONA de São Francisco de Paula, RS, Brasil
}

\author{
Ecological factors that determine the occurrence of Araucaria angustifolia and Podocarpus \\ lambertii in Mixed Ombrophylous Forest at São Francisco de Paula's FLONA, RS, Brazil
}

\author{
Solon Jonas Longhi* ${ }^{*}$ Doádi Antônio Brena ${ }^{\mathrm{I}}$ Sylviane Becker Ribeiro ${ }^{\mathrm{II}}$ \\ Cibele Rosa Gracioli ${ }^{\mathrm{II}}$ Régis Villanova Longhi ${ }^{\mathrm{IV}}$ Tarso Mastella $^{\mathrm{IV}}$
}

RESUMO

O presente trabalho foi realizado na Floresta Nacional (FLONA) de São Francisco de Paula, no Rio Grande do Sul. Teve como objetivo determinar, via análise de regressão logística, os fatores ambientais que influenciam a ocorrência das espécies Araucaria angustifolia (Bertol.) Kuntze e Podocarpus Iambertii Klotzsch ex Endl. em uma área de Floresta Ombrófila Mista na FLONA de São Francisco de Paula. Para o estudo, foram avaliados os indivíduos com $c a p=30 \mathrm{~cm}$, em 1.000 subunidades amostrais de $10 \times 10 \mathrm{~m}$, demarcadas em 10 conglomerados permanentes de 1 ha (100 $x 100 m)$ previamente instalados na floresta. Em cada subunidade amostral, foram avaliados os fatores passíveis de influenciar a ocorrência das espécies, como os fatores relativos ao habitat: físicos do solo (profundidade, presença de afloramentos rochosos e umidade), exposição à luz e inclinação do terreno; e os fatores relativos à concorrência: área basal, densidade do sub-bosque e frequência de indivíduos. Pelos resultados obtidos, foi possível concluir que a Araucaria angustifolia ocorre em locais com solos profundos, expostos para o norte e com baixa frequência de indivíduos. Por outro lado, Podocarpus lambertii prefere locais não pedregosos, pouco inclinados, com exposição sul, relativamente úmidos, com alta frequência de indivíduos e alta densidade do subbosque.

Palavras-chave: regressão logística, análise multivariada, floresta com araucária.

\section{ABSTRACT}

The present research was accomplished at São Francisco de Paula's National Forest (FLONA), in Rio Grande do Sul. The objective was to determine, through logistics regression analysis, the environmental and competition factors that could influence on the occurrence of Araucaria angustifolia (Bertol.) Kuntze and Podocarpus lambertii former Klotzsch Endl. species in Mixed ombrophylous forest. For the study, the individuals with cap $=30 \mathrm{~cm}$ had been evaluated, in 1000 samples of $10 \times 10 \mathrm{~m}$, demarcated in ten permanent conglomerate of 1 ha $(100 \times 100 \mathrm{~m})$ previously installed in the forest. In each sample the factors that might influence the occurrence of the species and the factors related the habitat was assessed as physical of the soil (depth, rocky outcrop and humidity), exhibition to the light and the relief inclination. The competition factors were: basal area, sub-forest density and individuals' frequency. For the obtained results it had been possible to conclude that the Araucaria angustifolia prefers places with deep soils, north exposition and low individuals frequency. On the other hand, Podocarpus lambertii prefers no stony places, little sloping, with south exposition, relatively humid, with high frequency of individuals and high sub-forest density.

Key words: logistics regression, multivariate analysis, araucaria's forest.

\section{INTRODUÇÃO}

O entendimento dos processos ecológicos e dinâmicos das populações vegetais são requisitos básicos que permitem subsidiar ações de conservação e manejo em áreas silvestres. $\mathrm{O}$ conhecimento das interações entre as florestas e o ambiente possibilita fazer inferências sobre as mudanças que ocorrem nos ecossistemas durante o tempo e são importantes informações para as atividades silviculturais e de manejo.

IDepartamento de Ciências Florestais, Centro de Ciências Rurais (CCR), Universidade Federal de Santa Maria (UFSM), 97105-900,

Santa Maria, RS, Brasil. E-mail: sjlonghi@smail.ufsm.br. *Autor para correspondência.

${ }^{\text {IIC }}$ urso de Engenharia Agrícola, Universidade Luterano do Brasil (ULBRA), Santarém, PA, Brasil.

IIIPrograma de Pós-graduação em Engenharia Florestal, CCR, UFSM, Santa Maria, RS, Brasil.

${ }^{\mathrm{IV}}$ Curso de Engenharia Florestal, CCR, UFSM, Santa Maria, RS, Brasil. 
Um dos principais fatores que atuam na composição florística e estrutura das florestas é a heterogeneidade ambiental, que é resultado da diversidade de fatores que interagem nas comunidades, e a resposta das espécies a esses fatores faz que cada local tenha características próprias e características que são comuns a outros locais, possibilitando observar tendência. Numa escala local, a topografia tem sido considerada como a mais importante variável na distribuição espacial e na estrutura das florestas tropicais, porque ela corresponde às mudanças nas propriedades do solo, particularmente no regime de água e na fertilidade (RODRIGUES et al., 2007).

Em estudo realizado em um fragmento de floresta semidecídua em Lavras, Minas Gerais (MG), pesquisadores constaram que a heterogeneidade ambiental do fragmento foi caracterizada principalmente pela topografia acidentada e pelas variações de fertilidade, granulometria e regime hídrico dos solos, sendo este último o mais fortemente correlacionado com a distribuição das espécies (SOUZA et al., 2003).

Métodos de regressão têm se tornado um componente integral de qualquer análise de dados relacionados com a descrição da relação entre uma variável resposta (dependente) e uma ou mais variáveis explanatórias (independentes). O modelo de regressão logística é adequado para estimar a probabilidade de um evento dicotômico ocorrer. A presença de uma espécie em uma área pode ser considerada como um fenômeno dicotômico. Existem somente duas hipóteses: ou a espécie ocorre ou não ocorre. A regressão logística pode, nesse caso, ser usada para descobrir as variáveis que influenciam a probabilidade de ocorrência de uma espécie (HOSMER \& LEMESHOW, 1989).

A interação de qualquer modelo apropriado requer que se tirem inferências práticas dos coeficientes estimados do modelo. Esses coeficientes para as variáveis independentes representam a inclinação ou a taxa de mudança da variável dependente, em função da modificação de uma unidade da variável independente. A formulação lógica do modelo geral, ou seja, a seleção das variáveis independentes é, pois, a base para a correta utilização da regressão logística (HOSMER \& LEMESHOW, 1989).

Uma das vantagens da regressão logística é que se necessita saber apenas se um evento ocorreu para então usar um valor dicotômico como variável dependente. A partir desse valor dicotômico, o procedimento prevê sua estimativa da probabilidade de que o evento ocorrerá ou não. Se a probabilidade prevista for maior que 0,50 , então a previsão será sim, caso contrário será não (HAIR et al., 2005).
O conhecimento dos fatores ambientais é importante para o entendimento da dinâmica das florestas e serve de subsídio para futuros planejamentos de manejo silvicultural dessas espécies. Dessa forma, procurou-se no presente trabalho avaliar, por meio de análise de regressão logística, os fatores possíveis de avaliação rápida diretamente na área que mais influenciam a ocorrência das espécies de Araucaria angustifolia e Podocarpus lambertii na Floresta Ombrófila Mista da FLONA de São Francisco de Paula, no Rio Grande do Sul (RS).

\section{MATERIAL E MÉTODOS}

O estudo foi realizado na Floresta Nacional de São Francisco de Paula (FLONA), RS, localizada no nordeste do Estado, região dos Campos de Cima da Serra, Serra Gaúcha. A região é uma das mais úmidas do Estado, com pluviosidade superior a $2.000 \mathrm{~mm}$ e com temperatura média anual de aproximadamente $14,5^{\circ} \mathrm{C}$. De acordo com a classificação de Köppen, o clima é do tipo “Cfb”, mesotérmico médio (MORENO, 1961).

A presente pesquisa integra o conjunto de estudos previstos no Projeto Ecológico de Longa Duração - PELD/CNPQ - "Conservação e Manejo Sustentável de Ecossistemas Florestais - Bioma Araucária e suas Transições", em andamento na Unidade Experimental de Pesquisa na FLONA, período de 1999 a 2009.

Para determinar os fatores ambientais responsáveis pela ocorrência das espécies de Araucaria angustifolia e Podocarpus lambertii na área de estudo, foi utilizada a regressão logística. Muitas são as variáveis que podem influenciar o estabelecimento das espécies numa determinada área, dentre elas, os fatores relativos ao habitat (ecológicos). Consideraram-se os fatores físicos do solo (profundidade, quantidade de pedra e umidade), exposição e inclinação do terreno; e fatores relativos à concorrência, como a área basal, a densidade do subbosque e a frequência de indivíduos. Essas variáveis foram avaliadas em cada uma das 1.000 subunidades amostrais de $10 \times 10 \mathrm{~m}$ demarcadas em 10 conglomerados permanentes de 1 ha $(100 \times 100 \mathrm{~m})$ instalados na floresta.

A inclinação do terreno foi avaliada em \%, a exposição do terreno, em radianos, a frequência de indivíduos, em valores absolutos (número de indivíduos), e a área basal, em $\mathrm{m}^{2} \mathrm{ha}^{-1}$, foi determinada para os indivíduos com cap $=30 \mathrm{~cm}$. Para detectar a influência do tipo qualitativo do solo (profundo, raso, hídrico, presença de afloramentos rochosos) e a densidade do sub-bosque foi utilizado o conceito de dummy variables, conforme HOSMER \& LEMESHOW (1989). O solo profundo e hídrico foi tomado como 
padrão para interpretar a influência dos outros tipos. Dessa maneira, sempre que a parcela apresentava solo raso (profundidade $<30 \mathrm{~cm}$ ), hídrico e afloramentos rochosos, o valor dessas variáveis foi considerado $1 \mathrm{e}$ aos outros, o valor 0 . Além disso, sempre que havia subbosque denso atribuía-se valor 1 e, ao contrário, 0 .

A partir dos dados levantados elaborou-se uma matriz de dados, tendo como variável dependente a presença da espécie (valor 1) ou ausência (valor 0) e, como variáveis independentes, os valores dos fatores ecológicos e de concorrência que foram levantados nas subunidades amostrais. A partir dessa matriz de dados, realizou-se a Análise de Regressão Logística com auxílio do Programa SPSS (Statistical Package for the Social Science), versão 13.1, para Windows. Para a seleção sequencial das variáveis, utilizou-se o método Forward Stepwise. Adotou-se o modelo geral da regressão logística, que, segundo HOSMER \&

LEMESHOW (1989), é Probabilidade (evento) $=\frac{1}{1+e^{-Z}}$,

em que $Z$ é a combinação linear das diferentes variáveis que possam influenciar a probabilidade de ocorrência das espécies, isto é, $\mathrm{Z}=\mathrm{B}_{0}+\mathrm{B}_{1} \mathrm{X}_{1}+\mathrm{B}_{2} \mathrm{X}_{2}+\ldots+\mathrm{B}_{n} \mathrm{X}$. Os coeficientes $\left(\mathrm{B}_{0}, \mathrm{~B}_{1}, \mathrm{~B}_{2}, \ldots, \mathrm{B}_{\mathrm{n}}\right)$ são medidas dâs variações na proporção das probabilidades, chamadas de razão de desigualdade. São expressos em logaritmos, precisando ser transformados de volta (antilogaritmo), de forma que seu efeito relativo sobre as probabilidades seja avaliado mais facilmente. Um coeficiente positivo aumenta a probabilidade, ao passo que um valor negativo diminui a probabilidade prevista (HAIR et al., 2005). Para o presente estudo, o valor de $Z$ foi: $Z=B 0$ $+B_{1} u+B_{2} i+B_{3} p s+B_{4} e+B_{5} q p+B_{6} d s+B_{7} g+B_{8} f q$, sendo: $B_{0}, B_{1}, B_{2}, B_{3}, B_{4}, B_{5}, B_{6}, B_{7}$ e $B_{8}$ os coeficientes estimados dos dados; $u=$ umidade do solo, $i=$ inclinação do terreno, $p s=$ profundidade do solo, $e=$ exposição, $q p=$ quantidade de pedras, $d s=$ densidade do sub-bosque, $g$ = área basal e $f q=$ frequência de indivíduos, as variáveis independentes; $e=$ a base do logaritmo natural, aproximadamente 2,718. Portanto, o modelo geral testado para explicar a ocorrência das espécies Araucaria angustifolia e Podocarpus lambertii foi:

$$
P=\frac{1}{1+e^{-\left(B_{0}+B_{1} u+B_{2} i+B_{3} p s+B_{4} e+B_{5} q p+B_{6} d s+B_{7} g+B_{8} f q\right)} .}
$$

Para avaliar o quanto o modelo é apropriado, fez-se a comparação da precisão do modelo com dados reais, por meio de uma tabela de classificação. Essa tabela não revela a distribuição das probabilidades estimadas para um fato em dois grupos. Para cada grupo estimado, todas as tabelas mostram se a probabilidade estimada é maior ou menor que 50\% (CALDATO, 1998).
Além da tabela de classificação, avaliou-se a adequação do ajuste do modelo estimado, pela observação da semelhança entre os dados amostrais e os previstos pelo modelo, por meio da estátistica -2LL (- 2 Logarithm of the Likelihood), que compara o modelo em teste como um "modelo perfeito". Um bom modelo é o que tem uma alta semelhança entre os dados e o resultado do modelo, o que leva um pequeno valor de -2LL. Quando o modelo descreve perfeitamente os dados, o valor da verossimilhança observada é um. Para testar a hipótese nula de que a verossimilhança observada não difere de um (ajuste perfeito), pode-se usar o valor de -2LL. Sob a hipótese nula, que o modelo se ajusta perfeitamente, -2LL tem uma distribuição quiquadrada com $N$-p graus de liberdade, em que $N$ é o número de casos e $p$ é o número de parâmetros estimados. Se o nível de significância for grande, maior que $5 \%$, por exemplo, não se pode rejeitar a hipótese de que o modelo é apropriado (HOSMER \& LEMESHOW, 1989). Além disso, utilizaram-se diversas medidas do tipo $\mathrm{R}^{2}$ para representar o ajuste geral do modelo, como as de Cox \& Snell e de Nagelkerke (HAIR et al., 2005).

A análise estatística utilizada no trabalho foi realizada por meio do Software Statistical Package for Social Sciences (SPSS), versão 13.0 for Windows.

\section{RESULTADOS E DISCUSSÃO}

Para Araucaria angustifolia (Pinheirobrasileiro), a área basal $(g$ ) foi a primeira variável selecionada para compor o modelo da regressão logística, por apresentar maior escore estatístico e alta significância. Nos passos seguintes do processo Forward Stepwise, foram selecionadas, pela ordem, as variáveis profundidade do solo ( $p s)$, frequência de indivíduos $(f q)$ e exposição do terreno $(e)$. Dessa forma, o modelo específico para a estimativa da probabilidade de ocorrência do pinheiro-brasileiro no local do estudo ficou resumido a $P_{\text {Araucaria angustifolia }}=\frac{1}{1+e^{-\left(B_{0}+B_{1} \cdot g+B_{2} \cdot p s+B_{3} \cdot f q+B_{4} \cdot e\right)}}$

Já para Podocarpus lambertii (Pinheirobravo), seis variáveis foram selecionadas. A primeira foi frequência de indivíduos $(f q)$, seguida, pela ordem, das variáveis: umidade do solo $(u)$, exposição do terreno (e), densidade do sub-bosque (ds), quantidade de pedras ( $q p$ ) e inclinação do terreno (i). Assim, o modelo específico para a estimativa da probabilidade de ocorrência do $\boldsymbol{P}$. lamberti ficou

$P_{\text {Podocarpus lambertii }}=\frac{1}{1+e^{-\left(B_{0}+B_{1} \cdot f q+B_{2} \cdot u+B_{3} \cdot e+B_{4} \cdot d s+B_{5} \cdot q p+B_{6} \cdot i\right)}}$. 
$\mathrm{Na}$ tabela de classificação da regressão logística para a variável dependente "presença de $\boldsymbol{A}$. angustifolia" (Tabela 1), pode-se verificar que as 455 parcelas sem a presença dessa espécie (valor 0) foram corretamente estimadas pelo modelo como não tendo a espécie. Similarmente, 204 parcelas com presença de P. lambertii (valor um) foram corretamente estimadas como tendo a espécie. A diagonal secundária da tabela mostra quantas parcelas foram incorretamente classificadas, ou seja, $(233+108=341)$. Das parcelas sem a presença da espécie, $80,8 \%$ foram corretamente classificadas e das parcelas com pelo menos uma árvore de A. angustifolia houve $46,7 \%$ de acertos. No total, $65,9 \%$ das parcelas foram corretamente classificadas. Para cada grupo estimado, a tabela mostra se a estimativa da probabilidade é maior ou menor que 50\%, mas não revela a distribuição da probabilidade. Por exemplo, não se pode dizer dos dados da tabela 1 se as 233 parcelas com "falsa presença de Araucária" tinham probabilidades estimadas perto de $50 \%$, ou uma probabilidade estimada mais baixa.

Pode-se verificar, pela tabela 1 , para $\boldsymbol{P}$. Iambertii, que as 906 parcelas sem a presença da espécie (valor 0) foram corretamente estimadas pelo modelo como não tendo a espécie. Similarmente, uma parcela com presença da espécie (valor um) foi corretamente estimada como tendo a espécie. Pela diagonal secundária da tabela, nota-se que $93(90+3)$ parcelas foram incorretamente classificadas. Das parcelas sem a presença da espécie, 99,7\% foram corretamente classificadas e das parcelas com pelo menos uma árvore de $\boldsymbol{P}$. lambertii 1,1\% foram corretamente classificadas. No total, $90,7 \%$ das parcelas foram corretamente classificadas. Outra forma de julgar a performance do modelo logístico é examinar o quanto

Tabela 1 - Classificação da regressão logística para a variável dependente Araucaria angustifolia e Podocarpus lambertii.

\begin{tabular}{lccc}
\hline & Estimada & $\begin{array}{c}\text { Porcentagem } \\
\text { correta (\%) }\end{array}$ \\
\hline Araucaria angustfolia & 0 & 1 & \\
Observada & & & \\
0 & 455 & 108 & 80,8 \\
1 & 233 & 204 & 46,7 \\
& & Total & 65,9 \\
Podocarpus lambertii & & & \\
Observada & & & 9,1 \\
0 & 906 & 3 & 90,7 \\
1 & 90 & 1 & \\
& & Total & \\
\hline
\end{tabular}

o modelo se ajusta aos dados. Isso pode ser feito por meio da verossimilhança (-2LL), cujos resultados dessa estatística estão na tabela 2.

Observa-se, para a A. angustifolia, que o valor da verossimilhança (-2LL) no processo inicial $\left(\mathrm{B}_{0}\right)$ foi de $1.370,376$ e para P. lambertii foi de 609,691 . A medida que a variável é selecionada nos diferentes passos e interações, o valor de -2LL diminui, e o valor do teste qui-quadrado (?2) aumenta, melhorando a precisão do modelo. No modelo de uma só variável, o valor de -2LL é reduzido a partir do valor do modelo base de $1.370,376$ para $1.277,769$, uma queda de 92,607 para A. angustifolia e de 609,691 para 589,590, para $\boldsymbol{P}$. lambertii. Para A. angustifolia, no quarto passo (modelo de quatro variáveis), a redução do valor de 2LL foi de 117,741, que corresponde ao qui-quadrado da melhora do modelo e, como foi altamente significante $(0,000)$, indica que ocorreu uma melhora significativa no modelo com a inclusão das quatro variáveis. Para $\boldsymbol{P}$. lambertii, na redução do valor no sexto passo (modelo de seis variáveis), o valor de -2LL foi de 99,782, que corresponde ao qui-quadrado da melhora do modelo. Como o valor foi altamente significante $(0,000)$, indica também que ocorreu uma melhora significativa no modelo com a inclusão da frequência de indivíduos $(f q)$, umidade do solo $(u)$, exposição do terreno $(e)$, densidade do sub-bosque (ds), quantidade de pedras (qp) e inclinação do terreno (i).

As medidas de $\mathrm{R}^{2}$ de Cox \& Snell e $\mathrm{R}^{2}$ de Nagalkerke para o modelo de quatro variáveis $(\boldsymbol{A}$. angustifolia) foram de 0,111 e 0,149, respectivamente, indicando que houve melhora no modelo com a inclusão das quatro variáveis, quando comparadas com modelo de uma só variável (passo 1), cujos valores foram de 0,088 e 0,119 (Tabela 2). As mesmas medidas para o modelo de seis variáveis (P. lambertii) foram de 0,095 e 0,208 , respectivamente, indicando que houve relativa melhora no modelo com a inclusão das seis variáveis, quando comparadas com modelo de uma só variável (passo 1), cujos valores foram 0,020 e 0,044 (Tabela 2).

A tabela 3 contém os coeficientes logísticos e as demais estatísticas da seleção das variáveis independentes do modelo, isto é, quando a área basal, profundidade do solo, frequência de indivíduos e exposição do terreno são incluídas no modelo para $\boldsymbol{A}$. angustifolia e, quando a frequência de indivíduos, umidade do solo, exposição do terreno, densidade do sub-bosque, quantidade de pedras e inclinação do terreno são incluídas no modelo para $\boldsymbol{P}$. lambertii.

Como os níveis de significância observadas dos coeficientes foram menores que 0,05 , essas variáveis foram significativas e devem ser mantidas. Assim, o modelo definitivo, para Araucaria 
Fatores ecológicos determinantes na ocorrência de Araucaria angustifolia e Podocarpus lambertii...

Tabela 2 - Estatística de seleção das variáveis independentes em cada passo do modelo Forward Stepwise para Araucaria angustifolia ${ }^{1}$ e Podocarpus lambertii ${ }^{2}$.

\begin{tabular}{|c|c|c|c|c|c|c|}
\hline Passos & $-2 \mathrm{LL}$ & Qui-quadrado $\mathrm{n}\left(\chi^{2}\right)$ & GL & Sig. & Cox \& Snell R ${ }^{2}$ & Nagalkerke $\mathrm{R}^{2}$ \\
\hline \multicolumn{7}{|c|}{ Araucaria angustifolia } \\
\hline 1 & $1277,769^{\mathrm{a}}$ & 92,607 & 1 & 0,000 & 0,088 & 0,119 \\
\hline 2 & $1265,262^{\mathrm{a}}$ & 105,114 & 2 & 0,000 & 0,100 & 0,143 \\
\hline 3 & $1258,739^{\mathrm{a}}$ & 111,637 & 3 & 0,000 & 0,106 & 0,142 \\
\hline 4 & $1252,635^{\mathrm{a}}$ & 117,741 & 4 & 0,000 & 0,111 & 0,149 \\
\hline Constante $\mathrm{B}_{0}$ & 1370,376 & & & & & \\
\hline \multicolumn{7}{|c|}{ Podocarpus lambertii } \\
\hline 1 & $589,590^{\mathrm{a}}$ & 20,101 & 1 & 0,000 & 0,020 & 0,044 \\
\hline 2 & $572,294^{\mathrm{b}}$ & 37,397 & 2 & 0,000 & 0,037 & 0,080 \\
\hline 3 & $555,017^{\mathrm{b}}$ & 54,674 & 3 & 0,000 & 0,053 & 0,117 \\
\hline 4 & $534,957^{\mathrm{c}}$ & 74,734 & 4 & 0,000 & 0,072 & 0,158 \\
\hline 5 & $515,627^{\mathrm{c}}$ & 94,064 & 5 & 0,000 & 0,090 & 0,197 \\
\hline 6 & $509,909^{\mathrm{c}}$ & 99,782 & 6 & 0,000 & 0,095 & 0,208 \\
\hline Constante $\mathrm{B}_{0}$ & 609,691 & & & & & \\
\hline
\end{tabular}

* Estimativa final na interação 4 e 6, com significância menor que 0,001 .

${ }^{1}$ área basal, profundidade do solo, frequência de indivíduos e exposição do terreno.

${ }^{2}$ regressão logística com frequência de indivíduos, umidade do solo, exposição do terreno, densidade do sub-bosque, quantidade de pedras e inclinação do terreno.

angustifolia e Podocarpus lambertii, respectivamente, pode ser escrito como segue:

$$
\begin{gathered}
P_{\text {Araucaria angutifolia }}=\frac{1}{1+e^{-(-1,621+2,932 g+0,649 p s-0,058 f q+0,105 e)}}, \\
P_{\text {Podocapus lambertii }}=\frac{1}{1+e^{-(-6,137+0,109 . f q-1,156 \cdot u+0,324 \cdot e+1,831 . d s-2,569 \cdot q p+0,038 i)}}
\end{gathered}
$$

Observa-se, de acordo com a tabela 3 , que a área basal das espécies atuou de forma positiva, ou seja, quando a área basal é alta, as chances de ocorrência da espécie aumentam. $\mathrm{O}$ resultado pode ser deduzido pela observação do $\operatorname{Exp}(\mathrm{B})$. Com o aumento de uma unidade da área basal, a chance de ocorrência de $\boldsymbol{A}$. angustifolia aumenta 18,773 vezes. Além disso, deve-se considerar que, quando essa espécie está presente, a área basal é alta justamente porque a presença dela ocasiona esse aumento, em razão das características dominantes (grandes diâmetros) da espécie.

A profundidade do solo e a exposição do terreno também atuaram de forma positiva na distribuição da A. angustifolia na área. Entretanto, os valores baixos dos coeficientes $B$ indicam que sua influência na ocorrência da espécie não é tão

Tabela 3 - Variáveis independentes selecionadas pela regressão logística pelo método Forward Stepwise para Araucaria angustifolia e

\begin{tabular}{|c|c|c|c|c|c|c|}
\hline Variáveis & $\mathrm{B}$ & E.P. & WALD & GL & Sig. & Exp. (B) \\
\hline \multicolumn{7}{|c|}{ Araucaria angustifolia } \\
\hline Área basal $(g)$ & 2,932 & 0,327 & 80,348 & 1 & 0,000 & 18,773 \\
\hline Profundidade do solo ( $p s$ ) & 0,649 & 0,173 & 14,059 & 1 & 0,000 & 1,913 \\
\hline Freqüência indivíduos $(f q)$ & $-0,058$ & 0,022 & 7,226 & 1 & 0,007 & 0,943 \\
\hline Exposição do terreno $(e)$ & 0,105 & 0,043 & 6,059 & 1 & 0,014 & 1,111 \\
\hline Constante & $-1,621$ & 0,252 & 41,256 & 1 & 0,000 & 0,198 \\
\hline \multicolumn{7}{|c|}{ Podocarpus lambertii } \\
\hline Freqüência de indivíduos $(f q)$ & 0,109 & 0,031 & 12,582 & 1 & 0,000 & 1,115 \\
\hline Umidade do solo $(u)$ & $-1,156$ & 0,279 & 17,104 & 1 & 0,000 & 0,315 \\
\hline Exposição do terreno $(e)$ & 0,324 & 0,084 & 15,066 & 1 & 0,000 & 1,383 \\
\hline Densidade do sub-bosque ( $d s)$ & 1,831 & 0,482 & 14,422 & 1 & 0,000 & 6,239 \\
\hline Quantidade de pedras ( $q p)$ & $-2,569$ & 0,747 & 11,839 & 1 & 0,001 & 0,077 \\
\hline Inclinação do terreno (i) & 0,038 & 0,015 & 6,237 & 1 & 0,013 & 1,039 \\
\hline Constante & $-6,137$ & 0,697 & 77,546 & 1 & 0,000 & 0,002 \\
\hline
\end{tabular}
Podocarpus lambertii. 
significativa, o que é comprovado também pelo baixo Exp (B). Com o aumento de uma unidade na profundidade do solo e na exposição do terreno, a chance de ocorrência da $\boldsymbol{A}$. angustifolia aumenta 1,913 e 1,111 vezes, respectivamente. Essa afirmação corrobora CARVALHO (2003), relatando que $\boldsymbol{A}$. angustifolia apresenta crescimento limitado em solos rasos. Também, segundo ZANON (2007), a espécie apresenta bom crescimento em solos bem estruturados, enquanto que, em solos neossolos litólicos e gleissolos (hidromórficos), em consequência das restrições físicas para o desenvolvimento radicial, produzem condições de crescimento extremamente pobres.

Com relação à exposição do terreno, observou-se, pelos dados coletados no campo, que a maioria das parcelas com ocorrência de $\boldsymbol{A}$. angustifolia (73\%) possui exposição entre o leste e oeste na direção norte, onde há maior incidência solar, o que favorece a regeneração natural da espécie, embora os níveis de luz que chegam ao solo sejam filtrados pelo dossel da floresta. Para DUARTE et al. (2002), a espécie não é estritamente heliófita e pioneira, mas seu desenvolvimento é facilitado em locais mais ensolarados.

A frequência de indivíduos é também importante na ocorrência de A. angustifolia na área, embora em menor proporção. Isso pode ser deduzido pela significância apresentada. Pela interpretação do Exp (B), as chances de ocorrência da espécie em locais de frequência alta são de 0,943 vezes. Pelo valor negativo do coeficiente $B$, pode-se dizer que a espécie prefere ocorrer em locais de baixa densidade de indivíduos, ou seja, em locais com relativa luminosidade.

Observa-se também que, dos fatores que influenciaram a presença de Podocarpus lambertii na Floresta Ombrófila Mista da FLONA de São Francisco de Paula, a densidade do sub-bosque atuou de forma positiva, ou seja, quando a densidade do sub-bosque é alta, as chances de ocorrência da espécie aumentam (Tabela 3). O valor relativamente alto do coeficiente B para a densidade do sub-bosque indica que sua influência para a presença da espécie é significante. $\mathrm{O}$ resultado pode ser deduzido pela observação do Exp (B). Com o aumento de uma unidade da frequência, a chance de ocorrência de $\boldsymbol{P}$. lambertii aumenta 6,239 vezes. Assim, deve-se considerar que a espécie ocorre onde a densidade do sub-bosque é relativamente alta.

A exposição do terreno, a frequência de indivíduos e a inclinação do terreno também atuaram de forma positiva na distribuição de $\boldsymbol{P}$. Iambertii. Entretanto, os valores baixos dos coeficientes B indicam que suas influências na ocorrência da espécie não são tão significantes, o que é comprovado também pelo baixo Exp (B). Com o aumento de uma unidade na exposição do terreno, na frequência de indivíduos e na inclinação do terreno, a chance de ocorrência da $\boldsymbol{P}$. lambertii aumenta 1,383, 1,115 e 1,039 vezes, respectivamente.

Em relação à exposição do terreno, observou-se, pelos dados coletados, que a maioria das parcelas (61,5\%) possui exposição entre o sul e o oeste, onde a incidência solar é menor. Isso, em conjunto com a alta frequência dos indivíduos das parcelas de ocorrência do P. lambertii, comprovam, segundo CARVALHO (2003), que a espécie tem características de secundária tardia ou clímax tolerante a sombra. A partir de observações feitas a campo, pode-se constatar que esta apresenta excelente regeneração natural em capoeirões e vegetação secundária mais evoluída.

A presença de afloramentos rochosos e a umidade são também importantes na ocorrência de $\boldsymbol{P}$. lambertii, embora em menor proporção. Isso pode ser deduzido pelas significâncias apresentadas e pelos valores negativos dos coeficientes B. Pela interpretação dos Exp (B), as chances de ocorrência de P. lambertii em locais com pedras são de 0,077 e, em locais úmidos, de 0,315 .

Como a espécie prefere locais não pedregosos, aliado ao fato de ocorrer também em local com baixa inclinação, pois $75,8 \%$ das parcelas ocorreram em locais planos ou de baixa declividade, pode-se dizer que esta tem certa exigência por solos com teores médios de umidade.

\section{CONCLUSÕES}

Araucaria angustifolia prefere ocorrer em locais com solos profundos, expostos para o norte, onde há maior incidência de luz e, também, em locais com menor número de indivíduos, estes de grandes dimensões. Podocarpus lambertii prefere locais não pedregosos, pouco inclinados, com exposição sul, relativamente úmidos, com alta frequência de indivíduos e alta densidade do sub-bosque, indicando que a espécie tem características de secundária tardia, tolerante a sombra.

\section{REFERÊNCIAS}

CALDATO, S.L. Dinâmica populacional da Ocotea porosa (Lauraceae) na floresta ombrófila mista em Caçador, SC. 1998. 89f. Dissertação (Mestrado em Engenharia Florestal) - Programa de Pós-graduação em Engenharia Florestal, Universidade Federal de Santa Maria, RS.

CARVALHO, P.E.R. Espécies arbóreas brasileiras. Brasília: EMBRAPA, 2003. 1039p. 
DUARTE, L.S. et al. Assessing of the role of light availability in the regeneration of Araucaria angustifolia (Araucariaceae). Australian Journal of Botany, Collingwood, v.50, p.741751, 2002. Disponível em: <http://cat.inist.fr/ ?aModele $=$ afficheN\&cpsidt $=14385681>$. Acesso em: 15 jul. 2008. doi:10.1071/BT02027.

HAIR, J.F. et al. Análise multivariada de dados. 5.ed. Porto Alegre: Bookman, 2005. 593p.

HOSMER, D.W.Jr.; LEMESHOW, S. Applied logistic regression. New York: Wiley, 1989. 382p.

RIBEIRO, S.B. Classificação e ordenação da comunidade arbórea da floresta ombrófila mista da FLONA de São Francisco de Paula, RS. 2004. 181f. Tese (Doutorado em Engenharia Florestal) - Programa de Pós-graduação em Engenharia Florestal, Universidade Federal de Santa Maria, RS.

RODRIGUES, L.A. et al. Efeitos de solos e topografia sobre a distribuição de espécies arbóreas em um fragmento de floresta estacional semidecidual, em Luminárias, MG. Revista Árvore, Viçosa, v.31, n.1, p.25-35, 2007. Disponível em: <http:// www.scielo.br/scielo.php?script $=$ sci_arttext\&pid $=$ S0100$67622007000100004 \& \operatorname{lng}=\mathrm{pt} \& \mathrm{nrm}=\mathrm{iso} \& \mathrm{t} \operatorname{lng}=\mathrm{pt}>$. Acesso em: 17 nov. 2008. doi: 10.1590/S0100-67622007000100004.

SOUZA, J.S. et al. Análise das variações florísticas e estruturais da comunidade arbórea de um fragmento de floresta semidecídua às margens do rio capivari, Lavras-MG. Revista Árvore, Viçosa, v.271, n.2, p.185-206, 2003. Disponível em: <http:// www.scielo.br/scielo.php?script $=$ sci_arttext\&pid $=$ S010067622003000200009>. Acesso em: 08 dez. 2008. doi: $10.1590 / \mathrm{S} 0100-67622003000200009$.

ZANON, M.L.B. Crescimento da Araucaria angustifolia (Bertol.) Kuntze diferenciado por dioicia. 2007. $107 \mathrm{f}$. Tese (Doutorado em Engenharia Florestal) - Programa de Pósgraduação em Engenharia Florestal, Universidade Federal de Santa Maria, RS. 